\title{
Integrating Factor Methods as Exponential Integrators
}

\author{
Borislav V. Minchev \\ Department of Mathematical Science, \\ NTNU, 7491 Trondheim, Norway \\ Borko.Minchev@ii.uib.no
}

\begin{abstract}
Recently a lot of effort has been placed in the construction and implementation of a class of methods called exponential integrators. These methods are preferable when one has to deal with stiff and highly oscillatory semilinear problems, which often arise after spatial discretization of Partial Differential Equations (PDEs). The main idea behind the methods is to use the exponential and some closely related functions inside the numerical scheme. In this note we show that the integrating factor methods, introduced by Lawson in 1967, are also examples of exponential integrators with very special structure for the related exponential functions. In order to prove this relation, we use the approach based on bi-coloured rooted trees and B-series. We also show under what conditions every bi-coloured rooted tree can be express as a linear combination of standard non-coloured rooted trees.
\end{abstract}

\section{Introduction}

Realistic models of many physical processes require effective numerical solvers for a special class of partial differential equations, which after semidiscretization in space can be written in the following form

$$
u^{\prime}=L u+N(u(t)), \quad u\left(t_{0}\right)=u_{0},
$$

where $u: \mathbb{R} \rightarrow \mathbb{R}^{d}, L \in \mathbb{R}^{d \times d}, N: \mathbb{R}^{d} \rightarrow \mathbb{R}^{d}$ and $d$ is a discretization parameter equal to the number of spatial grid points. Several interesting problems can be brought to this form. Examples are Allen-Cahn, Burgers, Cahn-Hilliard, Kuramoto-Sivashinsky, NavierStokes, Swift-Hohenberg, nonlinear Scrödinger equations. Typically the linear part of the problem will be stiff and the nonlinear part will be nonstiff. Many numerical integrators have been developed to overcome the phenomenon of stiffness. Exponential integrators was introduced in the early sixties as an alternative approach for solving 
stiff systems. The main idea behind these methods is to integrate exactly the linear part of the problem and then use an appropriate approximation of the nonlinear part. Thus the exponential function, and functions which are closely related to the exponential function, appear in the format of the method. This was the reason why, until recently, these methods have not been regarded as practical. The latest achievements in the field of computing approximations to the matrix exponential, have provided a new interest in the construction and implementation of exponential integrators $[2,3,6,7,9]$.

The main requirements imposed on the functions, which appear in the format of an exponential integrator are, to be analytic, map the spectrum of $L$ into a bounded region in $\mathbb{C}$ and can be computed exactly or up to arbitrary high order cheaply. Suppose that, for all $l \in \mathbb{N}$ and $\lambda \in \mathbb{R}$, the operators $\phi^{[l]}(\lambda): \mathbb{R}^{d \times d} \rightarrow \mathbb{R}^{d \times d}$ satisfy the above conditions and can be expanded in the form

$$
\phi^{[l]}(\lambda)(h L)=\sum_{j \geq 0} \phi_{j}^{[l]}(\lambda)(h L)^{j} .
$$

The $\phi^{[l]}$ functions, which are used in practice are associated with the so called Exponential Time Differencing methods [3,4,10,11], and can be written explicitly as

$$
\phi^{[l]}(\lambda)(h L)=(\lambda h L)^{-l}\left(e^{\lambda h L}-\sum_{k=0}^{l-1} \frac{(\lambda h L)^{k}}{k !}\right) .
$$

If $h$ represents the stepsize and $U_{i}$ denotes the internal stage approximation of the exact solution for $i=1,2, \ldots, s$ then the computations performed in step number $n$ of an exponential Runge-Kutta $(\mathrm{RK})$ method are related by the equations

$$
\begin{aligned}
U_{i} & =\sum_{j=1}^{s} \sum_{l=1}^{s} \alpha_{i j}^{[l]} \phi^{[l]}\left(c_{i}\right)(h L) h N\left(U_{j}\right)+e^{c_{i} h L} u_{n-1}, \\
u_{n} & =\sum_{j=1}^{s} \sum_{l=1}^{s} \beta_{j}^{[l]} \phi^{[l]}(1)(h L) h N\left(U_{j}\right)+e^{h L} u_{n-1},
\end{aligned}
$$

where $\alpha_{i j}^{[l]}$ and $\beta_{j}^{[l]}$ are the parameters of the method and the vector $c=\left(c_{1}, c_{2} \ldots, c_{s}\right)^{T}$ is the abscissae vector. If $\alpha_{i j}^{[l]}=0$ for all $j \geq i$ 
the method is explicit and implicit otherwise. Alternatively the computations performed in step number $n$ can be represented in a more Runge-Kutta type formulation as follows

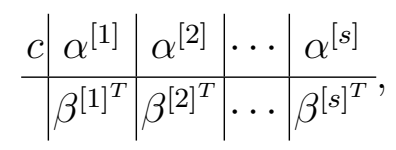

where each element in row number $i$ of the matrix $\alpha^{[l]}$ is multiplied by $\phi^{[l]}\left(c_{i}\right)(h L)$ and each element in the vector $\beta^{[l]^{T}}$ is multiplied by $\phi^{[l]}(1)(h L)$. The resulting matrices are then added in a component by component sense.

Other important class of methods which are also used for solving the semilinear problem (1.1) are the Integrating Factor (IF) methods. The idea behind these methods goes back to the work of Lawson [8]. He proposes to ameliorate the effect of the stiff linear part of equation (1.1) by using change of variables (also known as Lawson transformation),

$$
v(t)=e^{-t L} u(t)
$$

The initial value problem (1.1) written in the new variable is

$$
v^{\prime}(t)=e^{-t L} N\left(e^{t L} v(t)\right) \quad v\left(t_{0}\right)=v_{0},
$$

where $v_{0}=e^{-t_{0} L} u_{0}$. The approach now is to apply an arbitrary $s$ stage Runge-Kutta method to the transformed equation (1.4) and then to transform the result back into the original variable. Thus, a method which satisfy just the nonstiff order conditions will not suffer from sever order reduction, when it is applied to stiff problems.

The aim of this paper is to show that the IF methods are examples of exponential RK methods with special choices for the $\phi^{[l]}$ functions and the parameters $\alpha^{[l]}$ and $\beta^{[l]^{T}}$. We also prove that in this special case the nonstiff order theory for the exponential RK methods reduces to the classical Runge-Kutta order theory, which explains why the IF methods exhibit the expected order.

The paper is organized as follows: we briefly survey the nonstiff order theory for the exponential RK methods in Section 2. Next, in Section 3, we define the structure the matrices $\alpha^{[l]}$ and the vectors $\beta^{[l]^{T}}$ as well as the form of the functions $\phi^{[l]}$, which correspond to the IF methods. Finally, in Section 4, we conclude with several remarks and questions of future interest. 


\section{Nonstiff order conditions}

The nonstiff order theory for the exponential RK methods was first constructed in [4] and later developed in [11]. Here we follow the approach suggested in [9]. It is based on bi-coloured rooted trees and B-series. For those not familiar with these concepts we suggest the monographs $[1,5]$ for a complete treatment.

Let $2 \mathrm{~T}^{*}$ denote the set of all bi-coloured (black and white) rooted trees with the requirement that the valency of the white nodes is always one. This correspods to the fact that the first term on the right hand side of (1.1) is linear. Let $\emptyset$ represents the empty set which remains if the root of the one node tree $\bullet$ or $\circ$ is removed. The order of the tree $\tau \in 2 \mathrm{~T}^{*}$ is defined as the number of vertices in the tree, and it is denoted by $|\tau|$. The density $\gamma$ of the tree is defined as the product over all vertices of the order of the subtree rooted at that vertex. An exponential Runge-Kutta method with elementary weight function $a: 2 \mathrm{~T}^{*} \rightarrow \mathbb{R}$, has nonstiff order $p$, if for all $\tau \in 2 \mathrm{~T}^{*}$, such that $|\tau| \leq p, a(\tau)=1 / \gamma(\tau)$.

In order to give a practical representation of the elementary weight function $a$ of the numerical solution, it is convenient to introduce some notations. Let for $l=0,1, \ldots$ and $k=1, \ldots, m$ the $s \times s$ $\operatorname{matrix} \phi_{l}^{[k]}(c)=\operatorname{diag}\left(\phi_{l}^{[k]}\left(c_{1}\right), \ldots, \phi_{l}^{[k]}\left(c_{s}\right)\right)$ and $C=\operatorname{diag}\left(c_{1}, \ldots, c_{s}\right)$. Define

$$
\begin{aligned}
A^{[l]} & =\sum_{k=1}^{s} \phi_{l}^{[k]}(c) \alpha^{[k]}, \\
b^{[l]^{T}} & =\sum_{k=1}^{s} \phi_{l}^{[k]}(1) \beta^{[k]^{T}}, \\
C^{[l]} & =\frac{1}{(l+1) !} C^{l+1} .
\end{aligned}
$$

The elementary weight function $a$ of the numerical solution can be computed using the following non-recursive rule:

- Attach $b^{[j]^{T}}$ to the root black node.

- Attach $A^{[j]}$ to all remaining nonterminal black nodes.

- Attach $A^{[j]}$ e to all terminal black nodes.

- Attach $C^{[j]} e$ to all terminal white nodes.

- Attach $I$ to all remaining white nodes. 
The value $j$ is the number of white nodes directly below the corresponding node, $I$ is the $s \times s$ identity matrix and $e=(1, \ldots, 1)^{T}$. Now for each tree multiply from the root to the leaf as in the case for Runge-Kutta methods, then multiply these expressions in a component by component sense.

\section{$3 \quad$ IF methods as a special case}

Applying a standard $s$-stage Runge-Kutta method to the transformed equation (1.4) and then transforming back the result into the original variable, leads us to the following $\phi^{[l]}$ functions

$$
\phi^{[l]}(\lambda)(h L)=e^{\left(\lambda-c_{l}\right) h L} .
$$

Every IF method can be represented in the form (1.3) with $\phi^{[l]}$ functions given by (3.1) and with a special choice of the coefficient matrices $\alpha^{[l]}$ and the coefficient vectors $\beta^{[l]^{T}}$. This choice reduces the set of all order conditions to a set, which consists only of the order conditions corresponding to the black trees. To proof of this fact we need the following lemma.

Lemma 1. Let $t \in \mathbb{R} \backslash\{0,-1,-2, \ldots\}$, then for $j=0,1,2, \ldots$

$$
\sum_{k=0}^{j} \frac{(-1)^{k}}{k !(j-k) !} \frac{1}{(k+t)}=\frac{1}{t(t+1) \cdots(t+j)} .
$$

Proof. The proof of this statement is by induction on $j$.

The following theorem defines the structure of the matrices $\alpha^{[l]}$ and the vectors $\beta^{[l]^{T}}$ for the IF methods. With this structure of the coefficients, to achieve certain nonstiff order, it is sufficient to satisfy only the black trees. This implies that the transformed differential equation (1.4) is solved using a Runge-Kutta method.

Theorem 1. Let all the non-zero coefficients of an exponential RungeKutta method (1.3), with $\phi^{[l]}$ functions given by (3.1) be located in column number $l$ of the matrix $\alpha^{[l]}$ and in position number $l$ of the vector $\beta^{[l]^{T}}$ for $l=1,2, \ldots, s$. The method has nonstiff order $p$ iff all order conditions corresponding to the black trees are satisfied. 
Proof. It follows directly that if the exponential Runge-Kutta method has nonstiff order $p$ then all order conditions corresponding to the black trees are satisfied. Let us assume that all the order conditions corresponding to the black trees are satisfied. We need to prove that all the remaining order conditions are also satisfied. From the definition of the $\phi^{[l]}$ functions (3.1), it follows that for $j=0,1,2, \ldots$,

$$
\phi_{j}^{[l]}(1)=\frac{\left(1-c_{l}\right)^{j}}{j !}, \phi_{j}^{[l]}(c)=\frac{1}{j !} \operatorname{diag}\left(\left(c_{1}-c_{l}\right)^{j}, \ldots,\left(c_{s}-c_{l}\right)^{j}\right) \cdot(3
$$

Since all order conditions corresponding to the black trees involve only the coefficients $A^{[0]}, b^{[0]^{T}}$ and $c$, we need to express every other order condition in terms of these coefficients. Having in mind the special structure of the matrices $\alpha^{[l]}$ and the vectors $\beta^{[l]^{T}}$, after substituting (3.2) into (2.1), we obtain for $j=1,2, \ldots$,

$$
\begin{aligned}
A^{[j]} & =\sum_{k=0}^{j} \frac{(-1)^{k}}{k !(j-k) !} C^{[0]^{j-k}} A^{[0]} C^{[0]^{k}}, \\
b^{[j]^{T}} & =\sum_{k=0}^{j} \frac{(-1)^{k}}{k !(j-k) !} b^{[0]^{T}} C^{[0]^{k}} .
\end{aligned}
$$

From the fact that all order conditions corresponding to the black trees are satisfied, it follows that $A^{[0]}, b^{[0]^{T}}$ and $c$ form a Runge-Kutta method. Therefore,

$$
\begin{gathered}
C^{[0]} e=A^{[0]} e, \quad C^{[0]} \zeta=\left(A^{[0]} e\right) \zeta, \\
C^{[0]^{k}} \zeta=\left(A^{[0]} e\right) \ldots\left(A^{[0]} e\right) \zeta,
\end{gathered}
$$

where $\zeta$ is an arbitrary vector and the multiplications between the elements in the brackets are in a component by component sense.

Now, we are in a position to define a procedure which transforms every coloured tree $\tau$ into a linear combination of black trees of order at most $|\tau|$. Each tree $\tau$ can be decomposed as $\tau=\left(\tau_{b}, \tau_{j}, \tau_{t}\right)$, where $\tau_{b}$ is a coloured tree on the bottom with less number of white nodes than $\tau$; $\tau_{j}$ is tall white tree with $j \geq 1$ white nodes and $\tau_{t}$ is black tree on the top. First applying formula (3.3) and then (3.4), for the order condition corresponding to a tree $\tau$, we obtain the following three representations in terms of black trees or trees with 
less white vertices.

If $\tau_{t}=\emptyset$ then $\tau$ reduces to

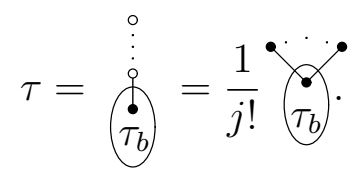

If $\tau_{b}=\emptyset$ then $\tau$ reduces to

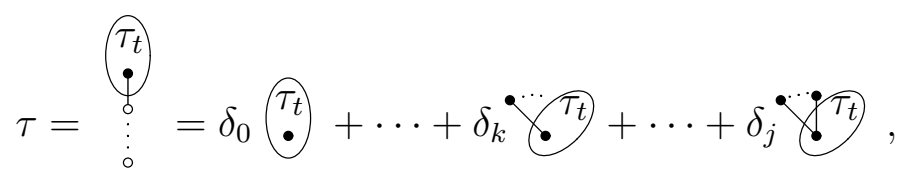

where $\delta_{k}=\frac{(-1)^{k}}{k !(j-k) !}$ for $k=0,1,2, \ldots, j$. In the general case when $\tau_{\{t, b\}} \neq \emptyset$, then $\tau$ can be represented as

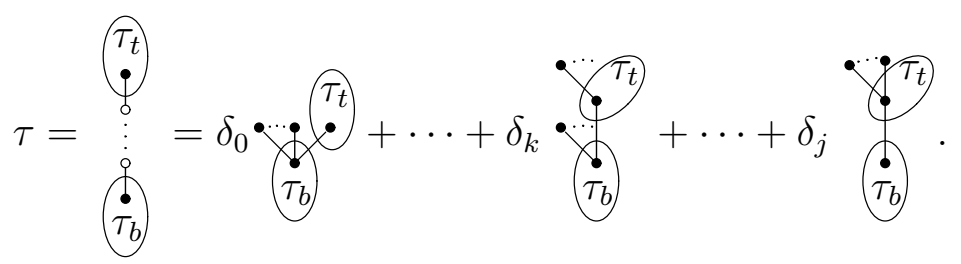

For each of the trees in the linear combination we apply the same procedure. Thus, after a finite number of steps all the trees in the combination will be black. From (2.1) it is clear that the order of every single black tree cannot exceed the order of coloured tree. To complete the proof we need to show that $a(\tau)=1 / \gamma(\tau)$ for all coloured trees $\tau$, where $|\tau| \leq p$. We prove this by induction on the number of steps $\theta$ in the transformation process. Let $\theta=1$. Every coloured tree $\tau$ has representation $\tau=\sum_{k=0}^{j} \delta_{k} \tau_{k}$, where all $\tau_{k}$ are black trees. If $\gamma\left(\tau_{t}\right)=x_{1}\left|\tau_{t}\right| x_{2}$ then $a\left(\tau_{k}\right)=\frac{1}{\gamma\left(\tau_{k}\right)}=\frac{1}{x_{1}\left(\left|\tau_{t}\right|+k\right) x_{2}}$ and by Lemma 1 for $t=\left|\tau_{t}\right|$ it follows that

$$
\begin{aligned}
a(\tau) & =\sum_{k=0}^{j} \frac{(-1)^{k}}{k !(j-k) !} a\left(\tau_{k}\right)=\sum_{k=0}^{j} \frac{(-1)^{k}}{k !(j-k) !} \frac{1}{x_{1}\left(\left|\tau_{t}\right|+k\right) x_{2}} \\
& =\frac{1}{x_{1}\left|\tau_{t}\right|\left(\left|\tau_{t}\right|+1\right) \cdots\left(\left|\tau_{t}\right|+j\right) x_{2}}=\frac{1}{\gamma(\tau)} .
\end{aligned}
$$

Assume that $a(\tau)=1 / \gamma(\tau)$ for all coloured trees $\tau$ with $\theta$ steps in the transformation process. Let $\tau$ be a tree with $\theta+1$ steps in the transformation process. From (3.5) it follows that $\tau=\sum_{k=0}^{j} \delta_{k} \tau_{k}$, 
where $\tau_{k}$ are coloured trees with $\theta$ steps in the transformation process and hence $a\left(\tau_{k}\right)=1 / \gamma\left(\tau_{k}\right)$. If $\gamma\left(\tau_{t}\right)=x_{1}\left|\tau_{t}\right| x_{2}$ then $a\left(\tau_{k}\right)=$ $\frac{1}{\gamma\left(\tau_{k}\right)}=\frac{1}{x_{1}\left(\left|\tau_{t}\right|+k\right) x_{2}}$ and by Lemma 1 for $t=\left|\tau_{t}\right|$ it again follows that $a(\tau)=1 / \gamma(\tau)$.

\section{Conclusions}

We have shown that the IF methods are examples of exponential Runge-Kutta methods with special structure of the coefficients matrices and the related $\phi^{[l]}$ functions. We have also proven that, in this special case, the nonstiff order theory for the exponential RK methods reduces to the classical Runge-Kutta order theory. This explains why the IF methods exhibit the expected order. Other examples of the $\phi^{[l]}$ functions, rather than (1.2) and (3.1), arise from the framework of Lie group methods see [9]. The question how to find the best set of $\phi^{[l]}$ functions is open and needs further investigation.

\section{References}

1. J. C. Butcher, Numerical methods for ordinary differential equations, John Wiley \& Sons, 2003.

2. M. Calvo and C. Palencia, A class of explicit multistep exponential integrators for semilinear problems, preprint, 2005.

3. P. M. Cox and P.C. Matthews, Exponential time differencing for stiff systems, J. Comput. Phys. 176 (2002), 430-455.

4. A. Friedli, Verallgemeinerte Runge-Kutta verfahren zur löesung steifer differentialgleichungssysteme, Lect. Notes Math. 631 (1978).

5. E. Hairer, C. Lubich, and G. Wanner, Geometric numerical integration, Springer, 2003, Number 31 in Springer Series in Computational Mathematics.

6. M. Hochbruck and A. Osterman, Explicit exponential Runge-Kutta methods for semilinear parabolic problems, Submitted to SIAM J. Numer. Anal., 2004.

7. Exponential Runge-Kutta methods for parabolic problems, To appear in Appl. Numer. Math., 2004.

8. J. Lawson, Generalized Runge-Kutta processes for stable systems with large Lipschitz constants, SIAM J. Numer. Anal. 4 (1969), 372-390.

9. B. Minchev, Exponential integrators for semilinear problems, Ph.D. thesis, University of Bergen, 2004.

10. S. Nørsett, An A-stable modification of the Adams-Bashforth methods, Lect. Notes Math. 109 (1969), 214-219.

11. K. Strehmel and R. Weiner, B-convergence results for linearly implicit one step methods, BIT 27 (1987), 264-281. 